\title{
Das Tecnologias e Sistemas de Informação à Proposta Tecnológica de um Sistema de Informação Para a Agroindústria: O Grupo Sousacamp
}

\author{
Frederico Branco ${ }^{1,2}$, José Martins ${ }^{1,2}$, Ramiro Gonçalves ${ }^{1,2}$ \\ fbranco@utad.pt,jmartins@utad.pt,ramiro@utad.pt \\ ${ }^{1}$ Universidade de Trás-os-Montes e Alto Douro, Vila Real, Portugal \\ ${ }^{2}$ INESC TEC e UTAD, Vila Real, Portugal \\ DOI: $10.17013 /$ risti.18.18-32
}

\begin{abstract}
Resumo: O setor agroindustrial tem crescido, a nível mundial, a um ritmo muito considerável ao longo dos últimos anos. Exemplo deste crescimento é o setor de produção de cogumelo, cuja especificidade do processo produtivo obriga a que os recursos empregues sejam otimizados e controlados ao mais ínfimo detalhe. Em virtude de a literatura não apresentar uma solução transversal para o setor de atividade referido, propusemo-nos a desenhar uma proposta tecnológica de um sistema de informação para o setor da produção de cogumelo. A proposta apresentada, composta por 13 blocos funcionais interligados, foi o resultado de uma análise crítica dos requisitos técnicos e funcionais do Grupo Sousacamp - um exemplo de organização pertencente ao setor de produção de cogumelo - à luz das teorias de adoção de tecnologias ao nível das organizações.
\end{abstract}

Palavras-chave: STI; Agroindústria; Agroalimentar; Adoção de Tecnologia.

From Information Systems and Technologies to a Technological Proposal for an Agri-Food Industry Information System: The Sousacamp Group

Abstract: The agri-food industry has been growing at a considerable pace over the recent years. An example of this growth is the mushroom production sector, whose specificities associated with the production process enforce a very controlled management of all the resources applied to that activity. Considering that the existing literature does not present a transverse solution to the referred activity sector, we have endured in an effort to present a technological proposal for an information system directed to the mushroom production sector. The presented proposal, composed by 13 interconnected functional blocks, is the result of a detailed analysis to the technical and functional requirements of Sousacamp Group - a leading organization from the mushroom production sector - merged with the arguments and perspectives presented by technology adoption theories.

Keywords: IST; Agri-food Industry; Agri-food; Technology Adoption. 


\section{Introdução}

A crescente utilização dos Sistemas e Tecnologias de Informação(STI) tem sido transversal a todas as indústrias. Um dos setores onde esta utilização se tem verificado com maior impacto, tanto ao nível do desempenho das organizações como na qualidade dos produtos e serviços prestados, tem sido o setor agroindustrial (Gebbers \& Adamchuk, 2010). Em países tipicamente reconhecidos como desenvolvidos, as organizações pertencentes ao referido setor de atividade fazem um uso contínuo de sistemas e tecnologias altamente sofisticadas com o objetivo de não só gerir o seu negócio, mas também de controlar e monitorizar permanentemente as suas produções, de forma a poderem obter a informação necessária para uma otimização de recursos e incremento produtivo (Lopes \& Melão, 2016; Sørensen et al., 2010; Sousa, Teixeira, \& Gonçalves, 2011).

Ainda que a realidade descrita represente, um importante passo na adequação de um setor de atividade a toda uma nova realidade social e económica, à luz da literatura científica analisada, os modelos de referência para arquiteturas de sistemas de informação associados à agroindústria não estão direcionados nem adequados às especificidades de todos os tipos de produção agrícola. Esta realidade é ainda mais premente quando direcionamos a nossa atenção para o setor de produção de cogumelos, cujos requisitos tornam a implementação de um sistema de informação, adequado e que permita ganhos de desempenho organizacional, bastante complexa (Branco, Gonçalves, Martins, \& Cota, 2015).

O sucesso de um Sistema de Informação (SI) depende, em grande parte, da arquitetura que o suporta. A arquitetura de um sistema de informação é relevante pois todos os SI estão inseridos num contexto, assim, as organizações assumem-se como entidades em constante mudança e evolução. Este processo de mudança está inerentemente dependente de um conhecimento da arquitetura que suporta cada um dos SI de uma organização, pois só assim poderemos antecipar os impactos negativos muitas vezes associados à mudança (Quartel, Steen, \& Lankhorst, 2012).

Tendo em conta o anteriormente mencionado como algo crítico para o sucesso das organizações do setor agroindustrial, em particular daquelas ligadas à produção de cogumelo, assumiu-se como base para o presente projeto de investigação a inexistência de uma arquitetura de sistema de informação direcionado a este setor. Desta forma, apresentamos neste artigo a base conceptual de uma proposta tecnológica direcionada ao setor, tendo por base o estudo de caso - o Grupo Sousacamp - como fonte de informação e orientações críticas para o desenho da proposta.

\subsection{Adoção de Sistemas e Tecnologias de Informação}

Os sistemas e tecnologias de informação são, de há muito a esta parte objeto de estudo por parte da comunidade académica e científica, cujo objetivo tem sido caracterizar verdadeiramente as suas limitações e virtudes, e também a forma como estas mesmas plataformas têm sido adotadas, tanto a nível individual como a nível organizacional (Aversano, Grasso, \& Tortorella, 2013; Branco et al., 2015). No que diz respeito ao âmbito do presente trabalho, iremos focar a nossa atenção na adoção das STI ao nível das organizações. 
A literatura científica existente indica que a adoção dos STI é um tema bastante descrito, sendo vários os modelos e teorias que visam caracterizar o referido processo de adoção (Petter, DeLone, \& McLean, 2013). Ainda assim, ao analisarmos de forma cautelosa a adoção de STI ao nível das organizações percebemos que a descrição deste processo de adoção não apresenta o mesmo nível de detalhe que o existente quando analisado ao nível individual.

De acordo com T. Oliveira and M. Martins (2011) e Martins, Gonçalves, Oliveira, Pereira, and Cota (2014), a teoria de Difusão da Inovação (DOI) apresentado por E. Rogers (2003), a framework Tecnologia-Organização-Ambiente (TOE) apresentada por Tornatzky and Fleischer (1990), a Teoria da Construção Institucional das Organizações de Scott and Christensen (1995) e o modelo de Iacovou, Benbasat, and Dexter (1995) são as principais teorias que caracterizam o processo de adoção de STI ao nível das organizações.

Com base em todos os trabalhos analisados, foi possível concluir que a construção de um SI que sirva de suporte ao negócio de um grupo económico, terá seguramente de ser feita tendo por base os padrões identificados pelos vários modelos e teorias de adoção de tecnologia existentes.

\section{Caracterização do Caso de Estudo - Grupo Sousacamp}

O Grupo Sousacamp é composto na sua maioria por empresas do setor agroalimentar, sendo a sua principal atividade a produção de cogumelos frescos (agaricus bisporus). O seu mercado principal é a Península Ibérica, encontrando-se, atualmente, em expansão para outras zonas geográficas, tais como: Norte de África e América Latina (Branco et al., 2015).

A primeira empresa do Grupo Sousacamp surgiu em 1989, na freguesia de Benlhevai, concelho de Vila Flor, dedicando-se à produção, comercialização e distribuição de cogumelos. A expansão do negócio, em 2007, esteve na génese do Grupo empresarial. A sua verticalização e diversificação culminaram na criação de múltiplas empresas e de novos centros de produção e transformação de cogumelos e de composto orgânico (Branco, 2014).

$\mathrm{Na}$ atualidade, a operação do Grupo contempla uma visão $360^{\circ}$ dos processos associados à produção de cogumelos, a saber: produção de substrato; produção, colheita e preparação de cogumelos; expedição e logística e, reutilização do substrato, como fertilizante e biomassa. A atividade é complementada pela comercialização de variadas espécies de cogumelos e hortícolas, produzidos por terceiros. O mercado do Grupo Sousacamp é composto pela sua presença em Portugal, onde é líder, e Espanha, onde tem uma quota de mercado expressiva. Adicionalmente, com uma posição menor, está presente nos mercados francês, alemão e holandês (Branco, 2014).

\section{Proposta Tecnológica de Suporte Funcional ao Grupo Sousacamp 3.1. O Processo de Adoção de Tecnologia}

É usualmente consensual reconhecer a influência direta dos STI no desempenho e posicionamento das organizações face à concorrência (Pereira, Martins, Gonçalves, \& Santos, 2014; Rijo, Varajão, \& Gonçalves, 2012). Devido ao forte crescimento, na última década, o Grupo Sousacamp tem vindo a alinhar o seu SI com a estratégia do negócio e 
modelo de governação. Este alinhamento pressupõe a correta adoção dos STI, indo ao encontro dos fatores que tradicionalmente a afetam.

Tendo em consideração que o objetivo principal de planeamento e implementação de um SI, fosse colateralmente acolhido e adotado por todo o Grupo, decidimos suportar a proposta tecnológica apresentada, nas várias teorias e modelos de adoção de tecnologia ao nível organizacional. Após análise detalhada, e à luz do apresentado por T. Oliveira and M. F. Martins (2011), todo o esforço de relacionamento das teorias de adoção de STI com o desenho de soluções tecnológicas, particularmente arquiteturas de SI, teve por base a teoria da Difusão da Inovação (E. M. Rogers, 2003) e a Framework TOE (Tornatzky \& Fleischer, 1990).

\subsubsection{Teoria da Difusão da Inovação (DOI)}

A DOI é uma teoria que procura explicar como, porquê e a que ritmo novas ideias e tecnologias se difundem pelas culturas. Segundo E. M. Rogers (2003) a difusão é um processo através do qual a inovação é transmitida por determinados canais ao longo do tempo para um conjunto de membros respeitantes a um sistema social.

Apesar da complexidade da teoria, esta identifica quatro componentes que afetam a difusão de uma nova ideia: 1) Inovação - A inovação é uma ideia, prática, ou objeto que é percebido como novo por um indivíduo ou outra unidade de adoção; 2) Canais de comunicação - são o meio pelos quais as mensagens passam de um indivíduo para outro; 3) Tempo - o período de inovação-decisão é o período de tempo necessário para passar pelo processo de inovação-decisão. A taxa de adoção é a celeridade relativa à qual uma inovação é adotada pelos membros de um sistema social; e 4) Sistema Social - é um agrupamento de unidades inter-relacionadas que estão implicadas na resolução de um problema para alcançar um objetivo comum.

Da decomposição da DOI, o processo de difusão da inovação está intrinsecamente subordinado ao capital humano envolvido no processo. Desta forma, a adoção tem de ser uma criação longitudinal a todo o sistema social para que a inovação possa persistir ao longo do tempo como algo frutuoso e usável (Bradford \& Florin, 2003).

No contexto das organizações as inovações são recorrentemente adotadas em função de dois tipos de inovação-decisão: decisões grupais de inovação e decisões de inovação fundamentadas no poder. O primeiro acontece quando a adoção de uma inovação é resultado de uma anuência entre os colaboradores de uma organização. O segundo surge quando a adoção de uma inovação é consequência da decisão de um conjunto reduzido de colaboradores com grande poder dentro da organização (E. M. Rogers, 2003). O processo de inovação na decisão na organização engloba colaboradores apelidados de paladino, que lideram a adoção de uma inovação e quebram os obstáculos que possam ocorrer à inovação (Bradford \& Florin, 2003).

Ainda de acordo com o definido na DOI, as caraterísticas individuais do líder da organização, as características internas da organização e a abertura do ecossistema em que a organização está localizada, são os contextos a ter em conta quando analisamos o processo de difusão da inovação. 


\subsubsection{Technology-Organization-Environment Framework (TOE)}

A Framework TOE, apresentado por Tornatzky and Fleischer (1990), ainda que visivelmente presente na literatura científica como uma teoria de adoção de tecnologia ao nível das organizações, é considerada por vários autores como sendo uma evolução da teoria da Difusão da Inovação. Considerada apropriada para analisar fatores contextuais que afetam a adoção de distintas inovações (Wu \& Subramaniam, 2009), vem sendo utilizada por vários autores para estudar a adoção de STI ao nível das organizações (Bosch-Rekveldt, Jongkind, Mooi, Bakker, \& Verbraeck, 2011; Low, Chen, \& Wu, 2011).

Segundo Hsu, Kraemer, and Dunkle (2006), o contributo da TOE vai além do auxílio na compreensão da adoção de tecnologia por parte das organizações, pois permite destacar a criticidade do meio envolvente à organização e dos relacionamentos que esta estabelece com esse mesmo meio envolvente. Desta forma podemos dizer que a framework TOE se apresenta como uma teoria que permite explicar três elementos do contexto organizacional que influenciam as decisões de adoção de tecnologia (Teo, Ranganathan, \& Dhaliwal, 2006): 1) Contexto tecnológico - abrange as tecnologias que são significativas para a organização. As tecnologias existentes são relevantes no processo de adoção, pois apoiam a definição dos limites e a cadência da alteração tecnológica que a organização consegue suportar. As tecnologias existentes, mas que ainda não são utilizadas, também afetam a inovação, quer exibindo o limite do que é exequível, quer exibindo à organização de que modo a tecnologia pode auxiliar a adaptar-se e a evoluir; 2) Contexto Organizacional - refere-se às propriedades e meios que a organização detém, abrangendo as estruturas de união entre os membros, o processo de comunicação dentro da organização, a dimensão da organização e o número de meios; e 3) Contexto Ambiental - diz respeito ao setor e âmbito de negócio, à existência de fornecedores de tecnologia e à regulação jurídica. Setores mais concorrenciais tendem a incitar a adoção da inovação. Setores em célere alargamento tendem a inovar mais rapidamente do que setores maduros.

\subsection{O Processo de Adoção de Tecnologia no Grupo Sousacamp}

O Grupo Sousacamp, como grupo empresarial em constante evolução, apresenta um padrão de adoção de inovações tecnológicas que se apresentam como algo complexo. Como forma de caracterizar o processo de difusão da inovação por todo o grupo, a equipa de investigação utilizou a teoria da Difusão da Inovação, fazendo um paralelo entre as variáveis independentes apresentadas na referida teoria e a realidade do Grupo. O tipo de inovação-decisão mais prominente é o modelo de governação autoritária em que a adoção e utilização de uma determinada inovação resulta da decisão de poucos colaboradores com grande autoridade. Esta realidade, ainda que não considerada do ponto de vista da gestão como a mais sensata, advém de uma centralização da decisão no fundador do Grupo e nos principais órgãos funcionais da empresa-mãe.

Apesar da atividade do Grupo Sousacamp estar centrada na indústria agroalimentar, que à partida num país como Portugal não é assumida como algo eminentemente tecnológica, a produção de cogumelos é um processo em que a componente tecnológica tem atualmente uma importância muito significativa. Em paralelo a esta realidade, o plano estratégico do Grupo visa um investimento em STI como veículo para incrementos 
em termos de competitividade e o uso eficiente de recursos. Esta dinâmica estimula assim os colaboradores a participarem no processo de inovação.

A decisão de adotar inovações é um processo mental ao qual um indivíduo é exposto. Esse processo inicia-se com a aquisição do conhecimento da inovação, seguido da formação de uma atitude no sentido da inovação, a decisão de adotar, ou não, a inovação, à implantação da nova ideia e, por fim, a confirmação da decisão de adoção da inovação (Huff \& McNaughton, 1991).

Segundo T. Oliveira and M. F. Martins (2011) e Branco (2014) a framework de adoção de tecnologia TOE inclui o contexto ambiental da organização, o que permite explicar melhor a adoção da inovação intraempresa, o que o converte num modelo mais completo. Com base neste pressuposto e naqueles mencionados anteriormente, levamos a cabo uma ação de matching entre aqueles que seriam os componentes do SI do Grupo Sousacamp e a Framework TOE (Figura 1). Esta ação e a respetiva distribuição dos referidos componentes pelos vários contextos apresentados pela TOE tiveram por base, um enquadramento específico, sendo este detalhe descrito individualmente de seguida.

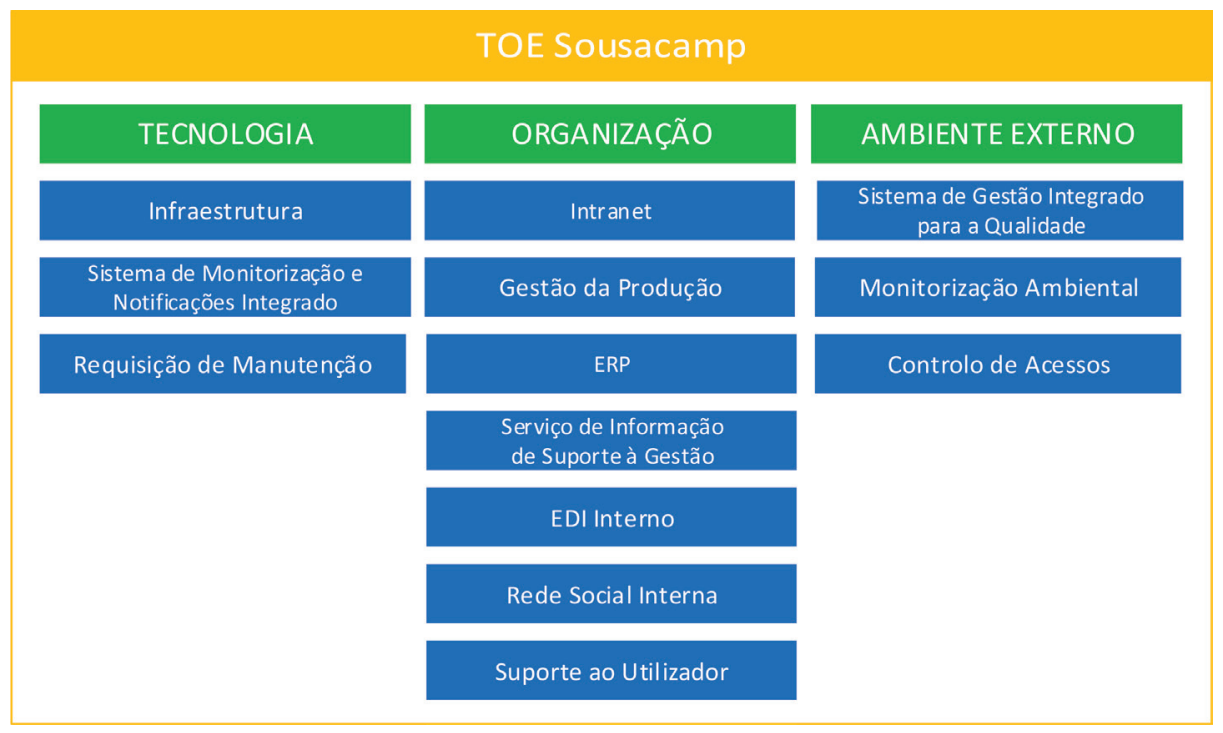

Figura 1 - Componentes do SI do Grupo Sousacamp analisados do ponto de vista da Framework TOE (Branco, 2014)

\subsection{Contexto Tecnologia 3.3.1. Infraestrutura}

A infraestrutura de uma organização deve ser pensada para que consiga suportar a homogeneidade das tecnologias existentes, garantido a centralização de conhecimento e constante diminuição da sofisticação das tecnologias de informação e comunicação utilizadas (Jansen, Brinkkemper, \& Finkelstein, 2013). Esta abordagem tende a agilizar a integração entre componentes do SI. 
A centralização de recursos relevantes fomenta uma gestão centralizada e, tendencialmente, uma redução de custos a toda a linha através da incorporação de tecnologias, como servidores virtuais, cuja execução não requer a presença física de especialistas. Desta forma, na presente proposta, a informação crítica encontra-se no data center da sede da empresa, o que potencia não só uma política otimizada de segurança, mas também uma recuperação de dados mais competente.

Em termos estruturais, a infraestrutura de telecomunicações foi desenhada em estrela e suportada por Virtual Private Network (VPN), criando assim uma rede privada transversal a todo o Grupo que permite não só a partilha horizontal de componentes por todas as empresas, numa lógica de SI global (verticalização da gestão do Grupo), como garante a escalabilidade do modelo.

\subsubsection{Sistema de Monitorização e Notificações Integrado}

O funcionamento otimizado de uma empresa obriga a um nível de preparação muito considerável por parte dos seus colaboradores, para que estes possam responder de forma célere a todos os possíveis eventos. Esta realidade é ainda mais crítica nas indústrias de produção contínua, onde a existência de impreparação ou a falta de capacidades de resolução de problemas podem levar a perdas muito significativas e impactos negativos relevantes (Gonçalves et al., 2014).

Uma análise ao Grupo Sousacamp revela a existência de vários sistemas cuja natureza obriga a uma constante monitorização e à existência de sistemas de notificação que possam ser executados na ocorrência de desvios a um, ou vários, padrões de funcionamento. Tal como foi possível constatar nas etapas iniciais de especificação da presente arquitetura, a existência de custos elevados de manutenção deve-se, em grande parte, à falta de informação atempada sobre o estado de falha de equipamentos críticos.

Tal como é mencionado por Branco (2014), "nas empresas do caso de estudo, é crítico garantir a climatização dos túneis de produção de substrato e das salas de produção de cogumelos, em particular, mas também monitorizar os sistemas complementares como, por exemplo, aqueles que compõem a cadeia de frio. Qualquer atraso na resposta a estas alterações pode invalidar o produto, tornando-o inútil”. Desta forma, a existência de um sistema de monitorização e notificação assume um papel crítico não só, na manutenção de um funcionamento mais linear do Grupo, como também, na redução de custos inerentes à resolução de problemas inerentes ao seu próprio funcionamento.

\subsubsection{Requisição de Manutenção}

A existência de um órgão funcional responsável pela manutenção, composto por pequenas equipas descentralizadas da sede do Grupo, forçou a criação de uma solução no âmbito dos SI que permitisse uma abordagem verticalizada de suporte ao processo de manutenção que fosse transversal a todo o Grupo.

Como solução para a necessidade de formalização e gestão de todos os pedidos de manutenção, foi adicionado um componente de requisição de manutenção à arquitetura proposta para que o SI possa apoiar toda a operacionalidade das ações de manutenção, principalmente, no que diz respeito ao seu registo e rastreabilidade. 


\subsection{Contexto Organização 3.4.1. Intranet}

Na última década o Grupo Sousacamp conseguiu atingir um nível de crescimento muito significativo não só em volume de negócio, como também em número de colaboradores e infraestrutura de suporte ao negócio, tendo este crescimento impactado de forma substancial a operação do Grupo. Em virtude deste facto, a proposta de arquitetura apresentada foi pensada englobando uma plataforma de desenvolvimento rápido de soluções que, de forma linear, permitisse que os especialistas do Departamento de Sistemas de Informação tivessem possibilidade de se focarem na componente funcional do negócio, em detrimento da componente tecnológica. Desta forma, fica assente que a manutenção de dados organizados e disponíveis transversalmente em todo o Grupo é um dos principais pilares do SI.

A necessidade de incrementar o desempenho dos processos de negócio do Grupo levou a que um conjunto de evoluções tivessem que ser definidas. Um exemplo destas evoluções é a implementação de um sistema de gestão documental que permita aos utilizadores trabalharem sobre os mesmos documentos de forma colaborativa e em paralelo, sendo o acesso a estes documentos gerido através de conjuntos de permissões atribuídas às bibliotecas de cada um dos departamentos e de cada um dos projetos. Em paralelo ao sistema de gestão documental foi também necessário definir um sistema de gestão de projetos que, de forma célere e eficiente, permitisse uma gestão e controlo das várias tarefas especificadas para cada um dos projetos, do nível de custos, qualidade, riscos, tempo, recursos humanos e materiais, e definir prioridades, controlando assim o estado de cada um.

De forma a garantir uma centralização de todos os sistemas mencionados, foi incorporada uma Intranet à proposta de arquitetura apresentada. Esta intranet é única e transversal a todo o Grupo. No que diz respeito às ferramentas colaborativas disponibilizadas, a Intranet do Grupo Sousacamp disponibiliza as seguintes: fóruns, wikis, blogs e questionários.

\subsubsection{Gestão da Produção}

Para controlar e tornar o mais eficiente possível a produção do Grupo, foi necessário implementar um conjunto de sistemas de controlo de automação, garantindo assim que todos os parâmetros inerentes ao processo produtivo (i.e.: temperatura, humidade, ventilação, etc.) são controlados em tempo real. Ainda que o referido processo produtivo incorpore bastantes automatismos, existem ainda várias fases da produção de cogumelo cuja realização é feita de forma manual.

Apesar do controlo do processo produtivo ser crítico, a sua gestão do ponto de vista funcional e administrativo também o é. Desta forma, a inexistência de um sistema de controlo de produção que permita, aos colabores do Grupo que realmente desempenham papeis de tomada de decisão, ter acesso a um conjunto de informações e dados de forma simples, direta e contextualizada, iria ter graves implicações para o negócio.

Tal como é argumentado por Branco (2014), nos últimos anos vários esforços têm sido desenvolvidos para a implementação de um sistema de suporte à produção totalmente ajustado às necessidades do Grupo Sousacamp. Este esforço advém principalmente do facto de as soluções existentes no mercado não se adequarem facilmente à realidade da produção intensiva de cogumelo e à dinâmica funcional e estrutural do Grupo. 


\subsubsection{ERP}

O histórico organizativo do Grupo Sousacamp, inicialmente meramente uma marca, sob a qual se enquadravam diversas empresas independentes, tinha a sua gestão de recursos feita através de vários Sistemas Integrados de Gestão (ERP) isolados em que cada um destes geria a informação de uma única empresa. A formalização do Grupo e a unificação das referidas empresas em torno do mesmo, tornou ainda mais evidentes as dificuldades inerentes a tarefas de migrações de dados e incorporação transversal de regras de negócio.

Tendo em conta que a mudança de software ERP acarreta um esforço financeiro e um conjunto de questões técnicas e funcionais de difícil exequibilidade, optou-se por adotar uma configuração do SI e do ERP que fosse capaz de responder ao seguinte conjunto de questões (Branco, 2014): “a) criação de normalizações ao nível dos códigos existentes, padronizando desta forma as várias entidades que se relacionam com o Grupo; b) imposição de uma política de preços transversal ao Grupo através de instituição de um mecanismo de aprovação de preços; c) garantir a rastreabilidade de propostas apresentadas aos clientes e definição de tabelas de preços de venda; d) garantir acesso aos colaboradores a funcionalidades referentes ao pedido de material, quando estes não possuem permissões diretas no ERP. Efetuar gestão dos pedidos, fomentando a agregação de pedidos e a eficiência na relação com os fornecedores; e) dispor de um serviço centralizado de análise de inventários que permita análises e correlações periódicas independentes de qual a empresa do Grupo que está a originar os dados; f) integrar um sistema de suporte à necessidade legal de controlo de assiduidade dos colaboradores, que seja capaz de gerir as suas entradas e saídas; e g) disponibilizar um repositório de contactos internos e externos ao Grupo".

\subsubsection{Serviço de Informação de Suporte à Gestão}

A necessidade de manutenção de elevados níveis de eficiência e competitividade fomentou a existência de um processo de tomada de decisão suportado por informação atual e de qualidade, independentemente do crescente número de fontes de dados.

Ainda que a existência de dados e informação com qualidade seja importante, a sua fácil utilização e interpretação torna o processo de decisão mais linear e incrementa a sua fiabilidade. Desta forma, e para que os colaboradores não fossem forçados a dominar ferramentas tecnológicas complexas, nem estivessem dependentes do departamento de TI para a criação de quadros de pilotagem personalizados, foi desenhado um sistema de Business Intelligence (BI) baseado numa abordagem de Self-service BI (SSBI). Esta ferramenta, que tem por base as tradicionais tecnologias de BI, incorpora ainda outras tecnologias que proporcionam aos utilizadores, sem conhecimentos técnicos em TI, a capacidade de tomarem decisões devidamente suportadas, sendo este processo de decisão enriquecido pelo contexto situacional, ou seja, os dados dizem respeito a um problema específico do negócio, que tipicamente têm um tempo de vida reduzido para um grupo de utilizadores específico (Turban, Sharda, Delen, \& Efraim, 2007).

\subsubsection{EDI Interno}

Mesmo com todo o esforço de adaptação do software às necessidades das empresas do Grupo, não é certo que todas as necessidades de cada empresa e dos vários departamentos 
sejam colmatadas, sendo este um fator que leva por vezes as empresas a adaptarem-se ao software ao invés de ser o inverso a acontecer.

De forma a ajustar o SI existente às questões associadas ao crescimento do Grupo, foi necessário incorporar na arquitetura de SI proposta um componente que tivesse a capacidade de otimizar a troca de documentos entre os vários atores e assim não só melhorar os tempos médios de inserção de dados, como também reduzir a taxa de erro associada à mesma.

Ainda que baseada numa implementação tradicional de um sistema EDI, o sistema desenvolvido no âmbito do Grupo Sousacamp assume como principais argumentos a seu favor os custos reduzidos, a segurança, o grau de adaptação ao processo de negócio e, principalmente, o seu nível de integração com o SI já implementado.

\subsubsection{Rede Social Interna}

A dispersão geográfica das empresas do Grupo Sousacamp, bem como a existência de competências funcionais em apenas algumas das empresas incentivou a procura por complementos ao SI existente que permitissem aos colaboradores da empresa interagirem entre si de forma colaborativa.

Desta forma, e seguindo as indicações de Branco (2014), para que uma rede social interna possa ser bem sucedida deve conseguir dar resposta aos seguintes requisitos funcionais: “a) Como proporcionar ao colaborador um local, gerido pelo próprio, que lhe permitisse agregar informação dispersa?; b) Como acompanhar num ponto central a atividade de colaboradores, serviços e projetos?; c) Como encontrar colaboradores com responsabilidades ou competências numa determinada área?; e d) Como saber, em tempo real, a disponibilidade dos colaboradores?”.

O componente Rede Social Interna foi desenvolvido como mecanismo de promoção da divulgação de conhecimento por todo o Grupo de forma mais informal. Para que esta ação seja o mais pessoal possível, todos os colaboradores possuem uma área individual (perfil), onde estão disponíveis os seus interesses e atividades. Tal como nas redes sociais tradicionais, as grandes vantagens da utilização deste tipo de tecnologia estão diretamente relacionadas com a possibilidade de informalmente podermos estabelecer relações com outros membros da rede (Martins et al., 2015).

\subsubsection{Suporte ao Utilizador}

Numa entidade que possui mais de 500 colaboradores uma das necessidades diárias é a existência de um serviço de suporte ao utilizador que não só seja tecnicamente capaz, mas que em paralelo esteja permanentemente disponível para resolver todos os problemas referentes aos equipamentos existentes.

Mantendo o alinhamento com a restante organização funcional, o serviço de suporte ao utilizador foi pensado para estar centralizado e ser disponibilizado transversalmente a todas as empresas do Grupo. A eficiência, critério muito relevante neste tipo de serviço, deve ser garantida através de uma formalização de todo o processo de suporte, desde a notificação da existência de um problema até à confirmação da resolução do mesmo. 
Desta forma, poderemos não só efetuar uma gestão clara de todo o procedimento como podemos inclusive garantir a necessária rastreabilidade do mesmo.

Em termos funcionais e tecnológicos o serviço de suporte ao utilizador (tipicamente denominados de help desk), fornece aos colaboradores do Grupo um contacto mais direto com os membros do departamento de TI responsáveis pelas atividades técnicas de suporte. Estes últimos são especialistas na área do suporte, sendo que o conhecimento necessário para executar as tarefas inerentes seja de difícil aquisição.

\subsection{Contexto Ambiente Externo 3.5.1. Sistema de Gestão Integrado para a Qualidade}

Ao longo da última década o investimento do Grupo Sousacamp na melhoria contínua nas suas atividades e processos de negócios (produtivos e administrativos) tem sido muito significativo, e tem resultado numa adoção de normas e standards internacionais. Esta adoção de certificações tem vindo a garantir a qualidade dos produtos e serviços, a garantir das condições de segurança alimentar e higiene e a preservação do meio ambiente envolvente.

Em termos práticos, para que seja possível manter um padrão de qualidade compatível com os standards e certificações internacionais, é necessário o desenvolvimento de um sistema de gestão da qualidade capaz de garantir que as atividades inerentes ao processo produtivo e administrativo são executadas corretamente e em linha com os critérios internacionais. Este sistema, cujas funcionalidades andam em torno da capacidade de tratar reclamações (não conformidades, ações preventivas, corretivas e oportunidades de melhoria), gestão de recursos hídricos e resíduos, legislação e controlo analítico e metrológico, visa acima de tudo uma otimização do funcionamento do Grupo e assim alcançar uma vantagem competitiva sobre a concorrência ao mesmo tempo que, ajuda a maximizar os lucros.

\subsubsection{Monitorização Ambiental}

A legislação em vigor direcionada à proteção ambiental e as exigências nesta mesma área apresentadas pelos standards e certificações internacionais, levou a que o Grupo tivesse de adotar um conjunto de boas práticas alinhadas com os seguintes requisitos (Branco, 2014): “a) Cumprimento legal e normativo (ISO 9001, ISO 22000 e ISO 14001); b) Melhoria da eficiência energética; c) Redução do impacto ambiental; d) Monitorização de sistemas de produção e apoio à produção; e) Suporte à manutenção preventiva dos equipamentos; f) Resposta rápida em caso de avarias; e g) Suporte à tomada de decisão".

Em termos práticos, o sistema de monitorização ambiental apresentado na atual proposta de arquitetura de SI é composto por uma rede de sensores sem fios, instalada em todas as empresas do Grupo, direcionada à medição constante de parâmetros como temperatura, humidade, luminosidade e energia elétrica consumida. Esta medição permite, a todo o momento, perceber qual o impacto ambiental que o processo produtivo está a ter e atingir os dados necessários para uma tomada de decisão informada e suportada perante as questões de natureza ambiental. 


\subsubsection{Controlo de Acessos}

A regulamentação existente para a área da segurança alimentar forçou a que o Grupo Sousacamp implementasse uma política de controlo de acessos às instalações de todas as empresas que o compõem.

De forma a tornar este controlo de acessos um processo menos burocrático, mais escalável e com uma maior capacidade de administração, o mesmo é feito através de equipas de segurança a que foi dado acesso ao SI do Grupo para que possam efetuar o registo de todas as entradas e saídas de pessoas e veículos. Este sistema de controlo de acesso pode ainda ser um complemento ao sistema de registo e assiduidade através da utilização de relógios de ponto e torniquetes.

\section{Análise e Discussão da Solução Proposta}

Para medrar é crítico inovar, pelo menos esporadicamente. No entanto, e apesar desta corroboração, foi árdua na esfera deste projeto de investigação, decidir que tecnologias adotar, quando as adotar e como governar o processo de implementação, de forma a produzir valor para o negócio. Muitos destes obstáculos deveram-se à complexidade do sistema e à mutação permanente de um conjunto alargado de variáveis de várias categorias, ao qual se agregou um alargado conjunto de premissas de racionabilidade sustentada numa fundamental análise custo-benefício.

\subsection{Implicações Teóricas e Práticas}

Observou-se que as tecnologias adotadas para cada contexto permitem um SI perfilado com um modelo de gestão verticalizado, com muitas das proficiências fundamentais centralizadas, mas suficientemente maleável para se adaptar a novas realidades. Estas respeitam modelos estáveis, ou seja, amplamente instituídos e com um extenso ecossistema de apoio, o que a largo prazo, concorre para a redução de dificuldades decorrentes de inconciliabilidades tecnológicas e funcionais.

As tecnologias adotadas são elementos facilitadores da adequada especificação de uma arquitetura de SI e do SI, avalizando estes o alinhamento com os requisitos do negócio, evitando desta forma prováveis quesitos que especificamente provêm da carência desse mesmo alinhamento.

Comprovou-se que a tecnologia adotada diminui o tempo empregue por parte dos recursos humanos habilitados nas tarefas de manutenção. A incorporação de sistemas de atualização automática ou com método de parametrização gráfica, simplifica e aprimora o processo de suporte, tornando-o mais eficiente. Deste modo, os colaboradores têm tempo acrescido para a concretização de atividades de maior retorno, o que se verte em níveis de desempenho superior com o consequente ganho de eficiência.

De uma perspetiva teórica o atual trabalho expõe como contributo mais pertinente a caracterização clara dos predicados intrínsecos a um SI de suporte ao setor de produção de cogumelo, podendo ser adaptado e customizado para outras atividades relacionadas com a indústria agroalimentar. No âmbito deste trabalho de investigação foi também possível, 
à luz da literatura científica e técnica existente, atingir os argumentos de sustentação necessários para que a proposta tecnológica apresentada possa ser considerada válida.

\section{Conclusões}

Ao longo dos últimos anos temos vindo a assistir a um crescimento muito significativo do setor agroindustrial, sendo muito desse crescimento acompanhado de uma cada vez maior utilização de sistemas e tecnologias de informação que suportam muitas das atividades inerentes não só à gestão das organizações, mas também ao controlo e maximização das próprias produções. Um exemplo claro deste crescimento muito sustentado na utilização de STI tem sido o setor da produção de cogumelo, cujas especificidades inerentes ao processo produtivo e logístico obrigam a um nível de controlo e eficiência de recursos muito preciso.

Ainda que a produção de cogumelo represente a nível mundial um valor económico já bastante considerável, não existia uma solução tecnológica transversal que se possa adequar e ajustar dinamicamente às organizações que têm por atividade a produção de cogumelo.

Com o presente trabalho propusemo-nos especificar uma proposta tecnológica para um sistema de informação que suportasse a atividade de uma organização produtora de cogumelo, sendo que para isso utilizámos o Grupo Sousacamp como caso de estudo. A proposta apresentada, composta por 13 blocos funcionais que se relacionam entre si de forma altamente interativa, foi desenhada de forma ponderada e com suporte nas várias teorias de adoção de tecnologia ao nível das organizações.

A nosso ver o presente trabalho representa não só um importante contributo não só para as organizações do setor da produção de cogumelo, mas também para a ciência pois ao longo da presente investigação são identificados um conjunto de requisitos e características inerentes à produção do referido fungo que podem ser utilizados em futuros trabalhos de investigação e desenvolvimento.

\section{Referências}

Aversano, L., Grasso, C., \& Tortorella, M. (2013). A literature review of Business/IT Alignment Strategies Enterprise Information Systems (pp. 471-488): Springer.

Bosch-Rekveldt, M., Jongkind, Y., Mooi, H., Bakker, H., \& Verbraeck, A. (2011). Grasping project complexity in large engineering projects: The TOE (Technical, Organizational and Environmental) framework. International Journal of Project Management, 29(6), 728-739.

Bradford, M., \& Florin, J. (2003). Examining the role of innovation diffusion factors on the implementation success of enterprise resource planning systems. International Journal of Accounting Information Systems, 4(3), 205-225.

Branco, F. (2014). Uma proposta de arquitetura de sistema de informação para as empresas agroalimentares do setor de produção de cogumelos: o caso Grupo Sousacamp. (PhD), University of Trás-os-Montes e Alto Douro. 
Branco, F., Gonçalves, R., Martins, J., \& Cota, M. (2015). Decision Support System for the Agri-food Sector - The Sousacamp Group Case. Paper presented at the World Conference on Information Systems and Technologies, Azores, Portugal.

Gebbers, R., \& Adamchuk, V. (2010). Precision agriculture and food security. Science, 327(5967), 828-831.

Gonçalves, R., Martins, J., Branco, F., Castro, M. R. G., Cota, M. P., \& Barroso, J. (2014). A new concept of 3D DCS interface application for industrial production console operators. UAIS.

Hsu, P.-F., Kraemer, K. L., \& Dunkle, D. (2006). Determinants of e-business use in US firms. International Journal of Electronic Commerce, 10(4), 9-45.

Huff, S. L., \& McNaughton, J. (1991). Diffusion of an Information Technology Innovation. The Business Quarterly, 56(1), 25-30.

Iacovou, C., Benbasat, I., \& Dexter, A. (1995). Electronic data interchange and small organizations: Adoption and impact of technology. MIS Quarterly, 19(4), 465-485.

Jansen, S., Brinkkemper, S., \& Finkelstein, A. (2013). Business network management as a survival. Software Ecosystems: Analyzing and Managing Business Networks in the Software Industry, 29.

Lopes, L. A., \& Melão, N. F. (2016). Website content and design in SME: insights from Portugal. International Journal of Electronic Business, 13(1), 70-97.

Low, C., Chen, Y., \& Wu, M. (2011). Understanding the determinants of cloud computing adoption. Industrial management \& data systems, 111(7), 1006-1023.

Martins, J., Gonçalves, R., Oliveira, T., Pereira, J., \& Cota, M. (2014). Social networks sites adoption at firm level: A literature review. Paper presented at the CISTI'2014 - Iberian Conference on Information Systems and Technologies, Barcelona, Spain.

Martins, J., Gonçalves, R., Santos, V., Cota, M. P., Oliveira, T., \& Branco, F. (2015). Proposta de um Modelo de e-Learning Social. RISTI-Revista Ibérica de Sistemas e Tecnologias de Informação(16), 92-107.

Oliveira, T., \& Martins, M. (2011). Literature review of information technology adoption models at firm level. The Electronic Journal of Information Systems Evaluation, 14(1), 110-121.

Oliveira, T., \& Martins, M. F. (2011). Literature Review of Information Technology Adoption Models at Firm Level. Electronic Journal of Information Systems Evaluation, 14(1), 110-121.

Pereira, J., Martins, J., Gonçalves, R., \& Santos, V. (2014). CRUDI Framework Proposal: Financial Industry Application. Behaviour \& Information Technology. doi:doi: 10.1080/0144929X.2014.914976

Petter, S., DeLone, W., \& McLean, E. (2013). Information Systems Success: The Quest for the Independent Variables. Journal of Management Information Systems, 29(4), 7-62. doi:10.2753/MISo742-1222290401 
Quartel, D., Steen, M. W., \& Lankhorst, M. M. (2012). Application and project portfolio valuation using enterprise architecture and business requirements modelling. Enterprise Information Systems, 6(2), 189-213.

Rijo, R., Varajão, J., \& Gonçalves, R. (2012). Contact center: information systems design. Journal of Intelligent Manufacturing, 23(3), 497-515.

Rogers, E. (2003). Diffusion of Innovations (5 Ed. ed.). New York: Free Press.

Rogers, E. M. (2003). Diffusion of Innovations, 5th Edition: Free Press.

Scott, W., \& Christensen, S. (1995). The institutional construction of organizations: International and longitudinal studies. Thousand Oaks, CA: Sage Publications.

Sørensen, C. G., Fountas, S., Nash, E., Pesonen, L., Bochtis, D., Pedersen, S. M., . . . Blackmore, S. (2010). Conceptual model of a future farm management information system. Computers and Electronics in Agriculture, 72(1), 37-47.

Sousa, S., Teixeira, M. S., \& Gonçalves, R. (2011). A aplicação do E-marketing em empresas de uma região Ultraperiférica: Região Autónoma da Madeira. RISTIRevista Ibérica de Sistemas e Tecnologias de Informação(7), 17-31.

Teo, T. S., Ranganathan, C., \& Dhaliwal, J. (2006). Key dimensions of inhibitors for the deployment of web-based business-to-business electronic commerce. Engineering Management, IEEE Transactions on, 53(3), 395-411.

Tornatzky, L., \& Fleischer, M. (1990). The processes of technological innovation: Lexington Books.

Turban, E., Sharda, R., Delen, D., \& Efraim, T. (2007). Decision support and business intelligence systems: Pearson Education India.

Wu, X., \& Subramaniam, C. (2009). Understanding RFID adoption in supply chain: An empirical study. Paper presented at the Proceedings of forty-second Hawaii international conference on systems science. 